\title{
COMPOSICIÓN DE LA DIETA DE CRAUGASTOR LINEATUS (ANURA: CRAUGASTORIDAE) DE CHIAPAS, MÉXICO
}

\author{
Matías Martínez-CoroneL ${ }^{1}$ y Marisol Pérez-Gutiérrez ${ }^{2}$ \\ ${ }^{1}$ Departamento de Biología, UAM-I. Av. San Rafael Atlixco 186, Col. Vicentina, CP. 09340, México, \\ D. F.<marti17@hotmail.com>. \\ ${ }^{2}$ Escuela de Biología, UNICACH. Tuxtla Gutiérrez, Chiapas, 29000.
}

Martínez-Coronel, M. \& M. Pérez-Gutiérrez. 2011. Composición de la dieta de Craugastor lineatus (Anura: Craugastoridae) de Chiapas, México. Acta Zoológica Mexicana (n. s.), 27(2): 215-230.

RESUMEN. Se describen los cambios ontogenéticos y estacionales de la dieta de Craugastor lineatus de Chiapas, México. De 54 contenidos estomacales de nueve juveniles, 38 machos y siete hembras, recuperamos 121 presas que representan 22 categorías de alimento. De estas, arañas, coleópteros, hormigas, isópodos, ortópteros, quilópodos y materia vegetal fueron las más importantes. El tamaño máximo de la presa estuvo relacionado positivamente con el tamaño de la rana, mientras que la relación con el número de presas fue negativa. Asimismo, los juveniles consumieron más coleópteros e isópodos que los adultos, quienes seleccionaron arañas, ortópteros y quilópodos. En la época húmeda encontramos 16 categorías de alimento, de las que ácaros, dípteros, isópteros y lepidópteros fueron exclusivas, mientras que en la época seca fueron 18 categorías, con diplópodos, fásmidos, odonatos, socópteros y seudoescorpiones como exclusivas.

Palabras clave: Craugastor lineatus, dieta, cambio ontogenético, cambio estacional, Chiapas.

Martínez-Coronel, M. \& M. Pérez-Gutiérrez. 2011. Diet composition of Craugastor lineatus (Anura: Craugastoridae) of Chiapas, Mexico. Acta Zoológica Mexicana (n. s.), 27(2): 215-230.

ABSTRACT. The ontogenetic and seasonal changes in the diet of Craugastor lineatus from Chiapas, México were described. We obtained 121 prey items from 54 stomachs by dissection, prey and prey parts were counted and identified to ordinal level. Twenty two food categories were identified. Frogs consumed mainly Aranae, Chilopoda, Coleoptera, Hymenoptera, Isopoda, Orthoptera, and plants, although the representation of each category varied ontogenetically and temporally. The maximum length of the prey items was positively correlated with frog body size; meanwhile, the number of prey items was negatively correlated with frog body size. Juveniles consumed more Coleoptera and Isopoda, while adults captured more Aranae, Orthoptera and Chilopoda. Sixteen items were consumed in wet season, with Diptera, Isoptera, Lepidoptera and Acari as exclusive. During the dry season were ingested 18 items, with Phasmatoidea, Odonata, Psocoptera, Diplopoda and Pseudoescorpionida as exclusive.

Key words: Craugastor lineatus, diet, onotogenetic change, seasonal change, Chiapas.

Recibido: 01/12/2008; aceptado: 22/02/2011. 


\section{INTRODUCCIÓN}

Los anuros postmetamórficos son depredadores importantes de artrópodos, además de otras categorías de alimento como crustáceos, moluscos, vertebrados y en ocasiones materia vegetal (Duellman \& Trueb 1994). Varios autores califican a las ranas como depredadores generalistas, por el hecho de consumir una variedad de presas, sin embargo, Toft (1981) considera que no ingieren indiscriminadamente cualquier organismo que este dentro de su espectro de aceptabilidad, por el contrario tienen preferencias por ciertas categorías, de manera que muchos de ellos pueden considerarse como especialistas. Diferentes factores influyen en la conformación de la dieta de un organismo, como son la morfología, el modo de forrajeo y la fisiología (Taigen \& Pough 1983, Simon \& Toft 1991). Asimismo, las condiciones climáticas estacionales, sobre todo el patrón de precipitación y la temperatura tienen un efecto importante en la dieta de las ranas, al afectar la diversidad y abundancia de las poblaciones de artrópodos de las cuales se alimentan estos animales (Donnelly 1991, Toft 1980b).

Debido a que los anfibios tragan entera a su presa, el tamaño y diseño de su aparato trófico los limita morfológicamente a explotar ciertas categorías alimentarias (Pianka 1978). En consecuencia, una presa de igual o mayor tamaño a las dimensiones del hocico puede resultar difícil de manejar y tragar, por el contrario una presa pequeña puede escapar fácilmente o no ser energéticamente rentable. Por esta razón, el tamaño de la rana se ha tomado como indicador del tamaño, tipo y número de presas que consume (Christian 1982, Donnelly 1991, Lima 1998, Maneyro et al. 2004). Asimismo, Toft (1981) y Simon \& Toft (1991) argumentan que el modo de forrajeo influye sobre el tipo y número de presas que una rana consume, y proponen que los organismos acechadores ("sit and wait") prefieren presas grandes y solitarias como arañas, coleópteros, ortópteros y lepidópteros, en comparación con las especies que son buscadoras activas ("widely foraging"), las que prefieren presas pequeñas y abundantes como ácaros y hormigas. En consecuencia, cabe esperar que las diferentes categorías de tamaño y edad que integran una población tengan dietas distintas. En este sentido, Lima \& Moreira (1993) encontraron que en Colostethus stepheni, un buscador activo, los juveniles consumieron principalmente ácaros y colémbolos (presas pequeñas), mientras que los adultos consumieron preferentemente formícidos y coleópteros (presas grandes). Resultados similares fueron hallados en Schoutedenella xenodactyloides (Blackburn \& Moreau 2006). Por otra parte, Duellman \& Lizana (1994) reportan que en Ceratophrys cornuta (un acechador), los juveniles consumieron mayor número de presas (principalmente formícidos) que los adultos (arañas, ranas, ratones y ortópteros).

Determinar la dieta de un organismo permite conocer las condiciones y recursos que la especie requiere para su supervivencia y en dado caso para su manejo, sobretodo en esta época de crisis ambiental. Asimismo, esta información es útil para entender mejor el papel que estos organismos desempeñan en el ecosistema del que forman 
parte. Por ejemplo, Beard et al. (2003) documentaron el efecto negativo que las ranas tienen sobre la abundancia de artrópodos herbívoros, actividad que disminuyó la tasa de herbivoría en el ecosistema a la vez que aumentó la producción del follaje de las plantas. Asimismo, Beard et al. (2002) determinaron el impacto positivo, que los productos de desecho y cadáveres de las ranas tuvieron en la disponibilidad de varios nutrientes que son limitantes de la productividad microbiana y de las plantas.

Craugastor lineatus (Brocchi 1879) se distribuye desde el este de Oaxaca y Chiapas, México, hasta las tierras altas del oeste de Guatemala, entre los 300- $2000 \mathrm{msnm}$ (Barrera et al. 2004), es un habitante de los bosques siempre verdes, con alto grado de humedad y sombra, en suelos con capas gruesas de hojarasca (Savage 1987). La biología e historia natural de esta especie es poco conocida, y en particular sobre su dieta. Recientemente sus poblaciones han disminuido dramáticamente por lo que fue catalogada como una especie amenazada por el libro rojo de la IUCN (Santos-Barrera et al. 2004). El presente estudio aporta información sobre la composición de la dieta y los cambios que ocurren durante el desarrollo ontogénetico y estacional en una población de esta especie, proveniente de un bosque mesófilo de Chiapas.

\section{ÁREA DE ESTUDIO}

El área de estudio está enclavada en la región fisiográfica Montañas del Norte de Chiapas, caracterizada por una topografía accidentada, formada por cerros altos y cañadas (95\%), con algunos sitios semiplanos (5\%) (De la Rosa et al 1989). El clima de la zona corresponde a un semicálido-húmedo, con temperatura máxima en mayo y mínima en enero, con temperatura media anual de $13.16^{\circ} \mathrm{C}$. La temporada de lluvia se presenta de junio a octubre, con una canícula marcada en agosto, la precipitación promedio es de $1978.7 \mathrm{~mm}$ (Zuill \& Lathrop 1975). El régimen de lluvias del área de estudio permitió diferenciar dos épocas a lo largo del año, una época húmeda, con precipitación abundante entre junio y octubre y una época con poca precipitación (aquí referida como "seca") que va de noviembre a mayo.

El trabajo se realizó en Río Negro, $4 \mathrm{Km}$ al norte de Coapilla $\left(17^{\circ} 10^{\prime} \mathrm{N}, 93^{\circ} 09^{\prime}\right.$ W), a $1700 \mathrm{~m} \mathrm{snm}$, Chiapas, México. La superficie muestreada fue de dos hectáreas y es atravesada por un arroyo permanente, conocido como "Río Negro", el cual tiene una anchura de 3-5 $\mathrm{m}$ y una profundidad máxima de $0.50 \mathrm{~m}$. El muestreo se llevo a cabo en dos tipos de vegetación, un bosque mesófilo y un pastizal cultivado. El bosque presentó árboles de Liquidambar styraciflua L., Quercus sp., Pinus sp. con aproximadamente los $30 \mathrm{~m}$ de altura, y un estrato medio de aproximadamente $5 \mathrm{~m}$ de altura, con Clusia flava Jacq. y helechos arborescentes en ambos márgenes del arroyo, y con un estrato arbustivo ralo de un metro de altura. Razón por la cual, el piso fue sombrío. La capa de hojarasca fue mayor a $5 \mathrm{~cm}$ de profundidad. El pastizal presentó pasto Pangola (Digitaria eriantha), bajo dos condiciones: un potrero en uso 
y uno abandonado. Un pastizal fue abandonado dos años antes del estudio (1993), y dominaron plantas de Rubus sp., Solanum sp., Pteridium sp. y árboles jóvenes de Liquidambar, que formaron un estrato de $2 \mathrm{~m}$ de altura, mientras que el pasto formo el estrato razante. El otro pastizal fue potrero y en él se encontraron individuos aislados de Pteridium sp. y Solanum sp.

\section{MÉTODOS}

Trabajo de campo. El material fue recolectado durante dos días de cada mes, de enero a diciembre de 1995, excepto en marzo, septiembre y noviembre. La captura de los ejemplares para determinar la dieta fue entre las 22:00 hrs y las 03:00 hrs del día siguiente. Asimismo, realizamos recorridos para determinar la actividad de las ranas en los dos tipos de vegetación, siguiendo transectos al azar y de longitud variable entre las 08:00-12:00 hrs y entre las 16:00-20:00 hrs. Muchos de los individuos fueron ubicados a través del canto, lo que suponemos resultó en un sesgo de machos en la proporción sexual. Los organismos fueron capturados manualmente y colocados en bolsas de manta por un periodo máximo de dos horas antes de darles muerte. Posteriormente fueron fijados con formol al $8 \%$ y conservados en alcohol al $70 \%$. De cada organismo recolectado registramos la fecha, hábitat, microhábitat, hora y estado del tiempo. Todos los ejemplares están depositados en la Colección Herpetológica del Museo Zoológico de la Universidad de Ciencias y Artes de Chiapas (MZ-ICACH). Trabajo de laboratorio. Las ranas fueron disectadas en el laboratorio y los contenidos estomacales examinados bajo el microscopio estereoscópico. El sexo de los organismos fue determinado con base en las gónadas y desarrollo del cuerpo graso, aquellos individuos donde no fue posible determinar si las gónadas eran ovarios o testículos las ubicamos como juveniles (Zug 1993). De cada rana medimos con un vernier, con aproximación de $0.01 \mathrm{~mm}$, la longitud hocico-cloaca (LHC), longitud de la tibia (LOTI), longitud del hocico (LOHO) y anchura del hocico (ANHO) de acuerdo con Duellman (2001). La categoría taxonómica que comprende el listado de presas corresponde al nivel de orden o clase (Chu 1949, Borror et al. 1989). Solo en himenópteros diferenciamos a formícidos de no-formícidos y en lepidópteros diferenciamos a las larvas de los adultos. El material que por su avanzado estado de descomposición no fue posible determinar, se considero bajo la categoría de artrópodos no determinados. De cada contenido estomacal contabilizamos las presas o partes de ellas y la categoría taxonómica; los restos vegetales fueron agrupados en una sola categoría. De todas las presas enteras medimos la longitud total (excluyendo patas y antenas) bajo un microscopio estereoscópico, con un vernier con aproximación de 0.01 $\mathrm{mm}$ y registramos el peso con una balanza analítica con aproximación de $0.001 \mathrm{~g}$. Análisis de la Información. Las diferencias morfométricas entre las tres categorías de sexo fueron evaluadas a través de un análisis de varianza. Para determinar la corre- 
lación entre las variables morfométricas de las ranas y evitar redundancia en los resultados usamos el índice de correlación de Pearson (Zar 1996), para elegir las variables que más información aportaban. Asimismo, mediante una prueba de chi cuadrada evaluamos el efecto del sexo y de la estacionalidad ambiental sobre la presencia de alimento en el estómago de las ranas (Zar 1996).

De cada categoría de alimento calculamos su valor de importancia (VI) de acuerdo con Bower et al. (1989), para lo cual aplicamos la siguiente fórmula: $\mathrm{VI}=\mathrm{Fi}+\mathrm{Pi}+\mathrm{Oi}$ donde:

$\mathrm{Fi}=$ Frecuencia relativa, es la proporción de individuos de una categoría de alimento respecto al total de todas las categorías.

$\mathrm{Pi}=$ Peso relativo, es la proporción del peso de una determinada categoría de alimento respecto al peso total de todas las categorías.

$\mathrm{Oi}=$ Ocurrencia relativa, es la probabilidad de encontrar una determinada categoría de alimento dentro de la muestra.

Los valores de VI suman 300, y los valores más altos fueron utilizados para evaluar las diferencias ontogenéticas y estacionales (época de lluvia y época seca) aplicando una prueba de $\mathrm{G}$ (Zar 1996).

El efecto del tamaño de la rana sobre el tamaño y el número de presas ingeridas fue evaluado con un análisis de regresión lineal. Finalmente la diversidad trófica o amplitud de nicho trófico fue estimada con el índice de Shannon-Wiener, y la similitud de la dieta fue estimada con el índice de Pianka (Krebs 1999).

\section{RESULTADOS}

Durante el periodo estudiado observamos 161 ejemplares de Craugastor lineatus activos sobre la hojarasca en el interior del bosque mesófilo: 37 fueron avistados entre las 08:00-12:00 hrs, 48 entre las 16:00-20:00 hrs y 76 entre las 22:00-03:00 hrs. Estos últimos fueron recolectados para caracterizar la dieta de la rana. La información antes mencionada mostró que $C$. lineatus esta activa las $24 \mathrm{hrs}$ del día, con preferencia por la tarde y noche.

De los 76 ejemplares recolectados durante la noche, 50 fueron machos, 10 hembras y 16 juveniles. La estructura poblacional de la muestra analizada estuvo sesgada hacia los machos, debido a que durante la búsqueda nocturna, el canto sirvió de guía para ubicarlos y capturarlos con mayor frecuencia.

Los resultados del análisis de correlación entre las cuatro variables morfométricas mostró un alto valor de dependencia entre la LHC y las otras variables (LOTI, $r^{2}=$ 0.97 , LOHO, $r^{2}=0.98$; ANHO, $r^{2}=0.95$ ), por lo que para evitar redundancia, solo utilizamos la primera de ellas para describir la variación del tamaño de la rana. El análisis de varianza mostró diferencias significativas $(F=86.89, p<0.0001)$ debidas al tamaño entre los tres grupos de sexo-edad considerados: las hembras fueron 
en promedio más grandes $(\mathrm{LHC}=37.02 \pm 1.24 \mathrm{~mm}$ desviación estándar y recorrido 28.46-41.99 mm) que los machos $(\mathrm{LHC}=26.68 \pm 0.55 \mathrm{~mm}, 19.2-36.4 \mathrm{~mm})$ y los juveniles $(\mathrm{LHC}=16.5 \pm 0.98 \mathrm{~mm}, 10.4-24.8 \mathrm{~mm}$ ). Con base en estos resultados, las tres categorías reconocidas previamente fueron mantenidas y usadas para describir los cambios de dieta ontogenéticos y estaciónales que más adelante presentamos.

Dieta general. De los 76 ejemplares capturados, 54 (71.05\%) tuvieron alimento, mientras que en 12 machos (15.78\%), tres hembras (3.94\%) y siete juveniles (9.21\%) el estómago estaba vacío. No encontramos evidencia que la falta de alimento estuviera influenciada por el sexo $\left(X^{2}=2.30\right.$, g.l. $\left.=2, p>0.05\right)$ o la estacionalidad $\left(X^{2}=\right.$ $0.54, g . l .=1, p>0.05)$.

En total determinamos 22 categorías de alimento, 21 de artrópodos y una de materia vegetal (Cuadro 1). El promedio de categorías por estómago fue de 2.05, con una desviación estándar 0.89. El número máximo de categorías de alimento en un estómago fue de 4. El promedio de presas por estómago fue de 2.16, con una desviación estándar de 1.09, el número máximo de presas en un estómago fue de 6. Las categorías con un VI $>15.00$ fueron ortópteros, coleópteros, arañas, quilópodos, isópodos, formícidos y materia vegetal. La diversidad trófica estimada en C. lineatus fue de 0.84 . Cambios ontogenéticos de la dieta. La dieta con la diversidad trófica más alta correspondió a las hembras $\left(H^{\prime}=0.95\right)$, quienes consumieron 10 categorías de alimento, de las que diplópodos y lepidópteros fueron exclusivos. Los machos y los juveniles tuvieron el mismo valor de diversidad $\left(H^{\prime}=0.88\right)$, pero divergieron en las categorías ingeridas. En los machos encontramos 18 categorías de alimento, de las cuales ácaros, dípteros, hemípteros, homópteros, larvas de lepidópteros, odonatos, socópteros y dictiópteros fueron ingeridos solo por ellos. Mientras que en los juveniles encontramos 10 categorías, con isópteros y seudoescorpiones como categorías exclusivas. Con referencia a las categorías compartidas, los coleópteros, formícidos e isópodos fueron ingeridos en mayor proporción por los juveniles, las arañas y ortópteros por los machos, en tanto que las hembras prefirieron a los ortópteros, quilópodos y materia vegetal (Cuadro 2).

Las diferencias de dieta entre las tres categorías de sexo-edad resultaron significativas $(G=385.54$, g.l. $=12, p<0.001)$. La mayor similitud trófica ocurrió entre machos y juveniles (Ojm $=0.69, G=168.07$, g.l. $=6, p<0.001)$ y entre machos y hembras $(\mathrm{Omh}=0.60, G=111.64$, g.l. $=6, p<0.001)$, mientras que el valor menor correspondió a las categorías de talla extrema, hembras y juveniles $(\mathrm{Ohj}=0.41, G=$ 282.29 , g.l. $=6, p<0.001$ ).

Al explorar la relación entre la talla de la rana y el tamaño y número de presas consumidas, empleamos la longitud del hocico (LOHO), ya que la cavidad oral es el sitio donde pasa el alimento que tragan estos animales, el tamaño del hocico debe ser una limitante para el manejo de la presa. La pendiente fue positiva entre LOHO de la rana y la longitud máxima de la presa $(\mathrm{LONGI})[\mathrm{LONGI}=(-4.13)+(1.28) \mathrm{LOHO}$, 
Cuadro 1. Composición de la dieta de Craugastor lineatus de Coapilla, Chiapas. Para cada categoría de alimento se da la frecuencia relativa $(\mathrm{Fi})$, porcentaje de frecuencia $(\% \mathrm{Fi})$, peso relativo $(\mathrm{Pi})$, porcentaje del peso $(\% \mathrm{Pi})$, ocurrencia relativa $(\mathrm{Oi})$, porcentaje de ocurrencia $(\% \mathrm{Oi})$ y valor de importancia (VI).

\begin{tabular}{|c|c|c|c|c|c|c|c|}
\hline Categoría & $\mathrm{F}$ & $\% \mathrm{~F}$ & $\mathrm{Pi}$ & $\% \mathrm{Pi}$ & I & $\% \mathrm{I}$ & VI \\
\hline \multicolumn{8}{|l|}{ Arachnida } \\
\hline Acari & 1 & 0.83 & 0.001 & 0.02 & 1 & 0.91 & 1.75 \\
\hline Araneae & 21 & 17.36 & 0.544 & 10.48 & 21 & 19.09 & 46.92 \\
\hline Pseudoescorpionida & 1 & 0.83 & 0.004 & 0.08 & 1 & 0.91 & 1.81 \\
\hline Chilopoda & 11 & 9.09 & 0.316 & 6.09 & 9 & 8.18 & 23.36 \\
\hline Diplopoda & 1 & 0.83 & 0.021 & 0.40 & 1 & 0.91 & 2.14 \\
\hline \multicolumn{8}{|l|}{ Crustacea } \\
\hline Isopoda & 11 & 9.09 & 0.151 & 2.91 & 8 & 7.27 & 19.27 \\
\hline \multicolumn{8}{|l|}{ Insecta } \\
\hline Coleoptera & 17 & 14.05 & 1.155 & 22.25 & 15 & 13.64 & 49.93 \\
\hline Diptera & 2 & 1.65 & 0.007 & 0.13 & 1 & 0.91 & 2.70 \\
\hline Dyctioptera & 3 & 2.48 & 0.149 & 2.87 & 2 & 1.82 & 7.17 \\
\hline Hemiptera & 2 & 1.65 & 0.046 & 0.89 & 2 & 1.82 & 4.36 \\
\hline Homoptera & 2 & 1.65 & 0.004 & 0.08 & 2 & 1.82 & 3.55 \\
\hline Hymenoptera (nf) & 3 & 2.48 & 0.139 & 2.68 & 3 & 2.73 & 7.88 \\
\hline Formicidae & 10 & 8.26 & 0.052 & 1.00 & 9 & 8.18 & 17.45 \\
\hline Isoptera & 1 & 0.83 & 0.003 & 0.06 & 1 & 0.91 & 1.79 \\
\hline Lepidoptera (A) & 1 & 0.83 & 0.01 & 0.19 & 1 & 0.91 & 1.93 \\
\hline Larvas & 3 & 2.48 & 0.482 & 9.28 & 3 & 2.73 & 14.49 \\
\hline Odonata & 1 & 0.83 & 0.018 & 0.35 & 1 & 0.91 & 2.08 \\
\hline Orthoptera & 13 & 10.74 & 1.89 & 36.40 & 12 & 10.91 & 58.06 \\
\hline Phasmantoidea & 2 & 1.65 & 0.074 & 1.43 & 2 & 1.82 & 4.90 \\
\hline Psocoptera & 1 & 0.83 & 0.002 & 0.04 & 1 & 0.91 & 1.77 \\
\hline Artrópodos no determinados & 6 & 4.96 & 0.02 & 0.39 & 6 & 5.45 & 10.80 \\
\hline Plantae & 8 & 6.61 & 0.104 & 2.00 & 8 & 7.27 & 15.89 \\
\hline TOTAL & 121 & 100 & 5.192 & 100 & & 99.99 & 300 \\
\hline
\end{tabular}

$\mathrm{nf}=$ no Formicidae, $\mathrm{A}=$ adultos.

$R^{2}=0.29 n=46, p<0.05$ ] (Figura 1), resultado que sugiere un mayor consumo de presas pequeñas $(x=4.55 \pm 0.26 \mathrm{~mm}$, recorrido 3.34-5.05 $\mathrm{mm})$ por los juveniles en comparación con los machos $(x=9.46 \pm 5.79 \mathrm{~mm}, 1.51-22.45 \mathrm{~mm})$ o las hembras 
Cuadro 2. Valores de importancia y de diversidad trófica $\left(\mathrm{H}^{\prime}\right)$ de las principales categorías de alimento encontradas en los tres grupos de sexo de Craugastor lineatus de Coapilla, Chiapas. La letra $n$ indica el tamaño de muestra de cada grupo.

\begin{tabular}{lccc}
\hline Categoría & Juveniles & Machos & Hembras \\
\cline { 2 - 4 } de alimento & $n=9$ & $n=38$ & $n=7$ \\
Araneae & 17.36 & 61.62 & 17.89 \\
Chilopoda & 9.27 & 18.86 & 70.29 \\
Isopoda & 32.66 & 18.58 & \\
Coleoptera & 153.31 & 23.32 & 19.35 \\
Formicidae & 26.36 & 17.59 & \\
Orthtoptera & 17.36 & 66.84 & 73.16 \\
Plantae & 17.36 & 13.12 & 25.85 \\
$\mathrm{H}^{\prime}$ & 0.88 & 0.88 & 0.95 \\
\hline
\end{tabular}

$(x=18.86 \pm 8.55 \mathrm{~mm}, 6.72-30.00 \mathrm{~mm})$. En cambio, la pendiente fue negativa entre LOHO y el número de presas ingeridas (PRESA) [PRESA $=(3.16)+(-0.08) \mathrm{LOHO}$, $\left.R^{2}=0.05, \mathrm{n}=54, p>.05\right]$ (Figura 2). Y no obstante que las diferencias no fueron estadísticamente significativas entre las tres categorías, la tendencia fue que los juveniles consumieron en promedio más presas $(x=2.77 \pm 1.39, n=9$, recorrido $2-6)$ en

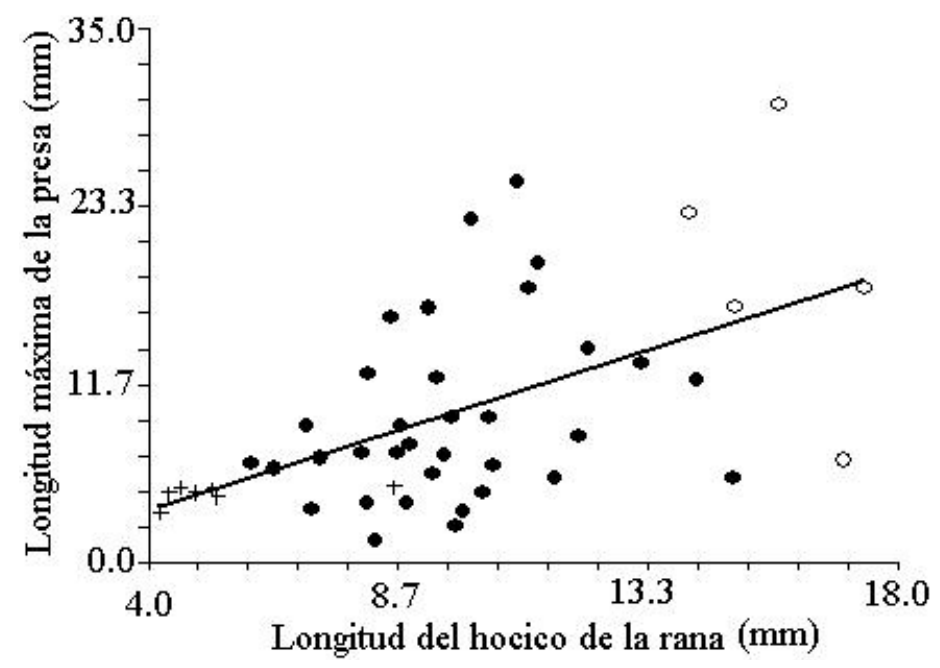

Figura 1. Relación entre la longitud del hocico $(\mathrm{mm})$ de Craugastor lineatus y la longitud máxima $(\mathrm{mm})$ de las presas ingeridas. La $(+)$ representa a los juveniles, el (•) a los machos y el (o) a las hembras. La línea continua representa la pendiente de regresión estimada. 


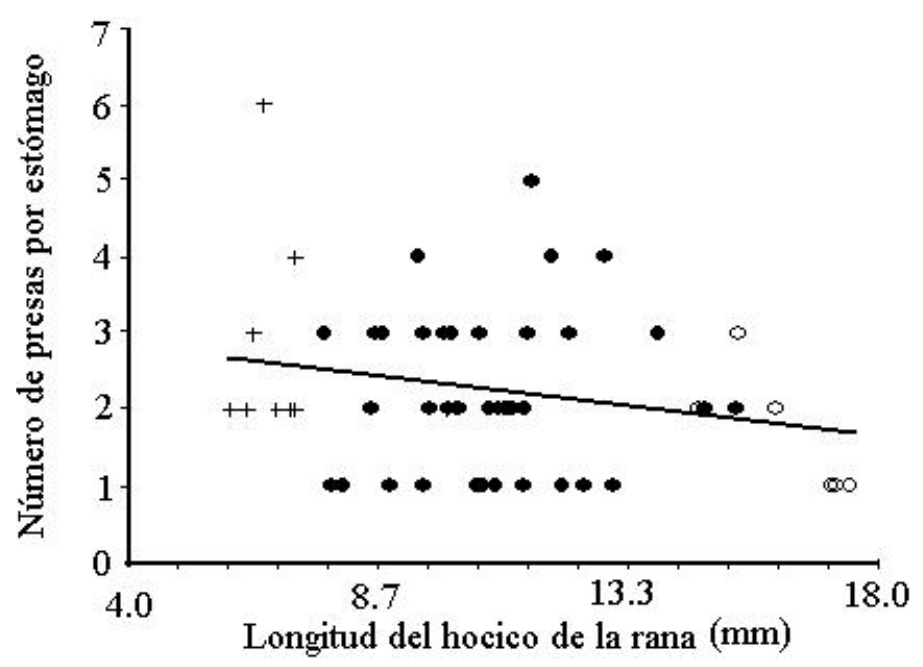

Figura 2. Relación entre la longitud del hocico de Craugastor lineatus y el número de presas ingeridas por individuo. $\mathrm{La}(+)$ representa a los juveniles, el (•) a los machos y el (o) a las hembras. La línea continua representa la pendiente de regresión estimada.

comparación con los machos $(x=2.21 \pm 1.04, \mathrm{n}=38,1-5)$ o las hembras $(x=1.71 \pm$ $0.75, n=7,1-3)$.

Cambios estacionales de la dieta. De las 22 categorías alimentarias encontradas en los estómagos de C. lineatus, 16 los hallamos en la época húmeda y 18 en la época seca. De estas categorías, los ácaros, dípteros, isópteros y lepidópteros fueron consumidos solo durante la época húmeda, mientras que diplópodos, fásmidos, himenópteros, odonatos, seudoescorpiones y socópteros lo fueron en la época seca. La diversidad trófica fue mayor en la época húmeda en comparación con la seca, y en ambas las hembras tuvieron la mayor amplitud de dieta, mientras que la menor correspondió a los machos de la época seca (Cuadro 3). Las diferencias estaciónales resultaron significativas tanto para las tres categorías de sexo analizadas en conjunto $(G=46.72$, g.l. $=6, p<0.001$ ), como en las comparaciones pareadas (Cuadro 4). En cuanto a la sobreposición de dieta, el valor mayor correspondió a los juveniles y el menor a las hembras. Mientras que por época, la mayor sobreposición de dieta ocurrió durante la época húmeda. Asimismo observamos que la menor similitud fue para las categorías de talla extrema, hembras y juveniles.

\section{DISCUSIÓN}

Los individuos de Craugastor lineatus fueron observados siempre en el piso del bosque mesófilo y en ninguna ocasión los encontramos en los pastizales. Estas observa- 
Martinez-Coronel \& Pérez-Gutiérrez: Dieta de Craugastor lineatus

Cuadro 3. Valores de importancia de las principales categorías de alimento y diversidad trófica $\left(\mathrm{H}^{\prime}\right)$ encontradas en cada grupo de sexo y por época del año de Craugastor lineatus de Coapilla, Chiapas.

\begin{tabular}{lcccccc}
\hline \multirow{2}{*}{$\begin{array}{l}\text { Categoría } \\
\text { de Alimento }\end{array}$} & \multicolumn{3}{c}{ Época Húmeda } & \multicolumn{3}{c}{ Época Seca } \\
\cline { 2 - 7 } & Juveniles & Machos & Hembras & Juveniles & Machos & Hembras \\
\hline Araneae & 18.53 & 36.95 & 41.70 & 17.64 & 76.49 & \\
Chilopoda & 19.11 & 18.68 & 58.55 & & 19.32 & 82.04 \\
Isopoda & 45.87 & 33.73 & & 54.74 & 10.62 & \\
Coleoptera & 142.38 & 22.11 & & 110.50 & 25.10 & 38.37 \\
Formicidae & 18.53 & 30.05 & & 41.73 & 9.48 & \\
Orthoptera & 18.53 & 9.99 & 117.90 & 17.64 & 90.93 & \\
Plantae & 18.43 & 25.60 & & 19.25 & 4.53 & 62.04 \\
H' $^{\prime}$ & 0.93 & 0.94 & 1.00 & 0.88 & 0.82 & 0.97 \\
\hline
\end{tabular}

ciones concuerdan con Savage (1987), quien registró a esta rana en zonas con alto grado de humedad y sombra, en suelos con capas gruesas de hojarasca, condiciones similares a las encontradas en el bosque mesófilo de Río Negro. La ausencia de esta rana en el pastizal, probablemente sea consecuencia de los cambios bruscos de insolación y humedad que deben experimentar estos ambientes al faltar el estrato arbóreo. Asimismo, la presencia del ganado en el potrero representa un peligro potencial para estas ranas, ante el peligro de morir aplastadas o bien no encontrar refugio en un suelo carente de una capa de hojarasca, el cual además esta sometido a compactación constante debido al pisoteo de los animales.

Savage (1987) menciona que $C$. lineatus es una especie que carece de cuerdas vocales y por lo tanto es muda. Sin embargo, cuando buscábamos a estos animales durante la noche, escuchamos en ocasiones un silbido simple y suave, que al buscar su procedencia siempre nos llevó a un ejemplar de esta especie.

Debido a que el balance energético es crucial para la supervivencia de cualquier organismo, Huey et al. (2001) exploraron la influencia del hábitat, ritmo de actividad, posición trófica, modo de forrajeo, especialización, tamaño del cuerpo y filogenia en el éxito del forrajeo en lagartijas y encontraron que las razones son variadas para cada grupo. No existe un estudio similar para anfibios, pero en el caso de $C$. lineatus no encontramos evidencia que la falta de alimento tuviese relación con el sexo del individuo o con la estacionalidad ambiental, de manera que habrá que explorar los otros factores. El porcentaje de individuos de $C$. lineatus sin alimento (28.95\%) fue alto en comparación con el valor reportado para otras especies de ranas que explotan hábitat similares como Eleutherodactylus johnstonei en la cual fue de 19.3\% (Ovaska1991), en Ceratophrys cornuta fue de $15.27 \%$ (Duellman \& Lizana, 1994), mientras que para Proceratophrys appendiculata (Boquimpani-Freitas et al. 2002) y Schoutede- 
Cuadro 4. Resultados de sobreposición de la dieta entre grupos de sexo y épocas del año en Craugastor lineatus de Coapilla, Chiapas. La J identifica a los juveniles, la $\mathrm{M}$ a los machos, la $\mathrm{H}$ a las hembras, la h a la época húmeda y la s a la época seca, Ojk es el valor del índice de Pianka, G corresponde al valor del estadístico G, g.l. representa los grados de libertad y P la probabilidad estadística a la que fue significativo el valor obtenido de $\mathrm{G}$.

\begin{tabular}{ccccc}
\hline Grupos comparados & Ojk & G & g.l. & P \\
\hline Mh*Ms & 0.65 & 120.61 & 6 & $<0.001$ \\
Mh*Hh & 0.46 & 279.33 & 6 & $<0.001$ \\
Mh*Hs & 0.37 & 216.47 & 6 & $<0.001$ \\
Mh*Jh & 0.74 & 88.88 & 6 & $<0.001$ \\
Mh*Js & 0.62 & 90.85 & 6 & $<0.001$ \\
Ms*Hh & 0.68 & 102.77 & 6 & $<0.001$ \\
Ms*Hs & 0.32 & 356.74 & 6 & $<0.001$ \\
Ms*Jh & 0.57 & 212.63 & 6 & $<0.001$ \\
Ms*Js & 0.52 & 241.75 & 6 & $<0.001$ \\
Hh*Hs & 0.29 & 361.20 & 6 & $<0.001$ \\
Hh*Jh & 0.27 & 415.04 & 6 & $<0.001$ \\
Hh*Js & 0.22 & 483.97 & 6 & $<0.001$ \\
Hs*Jh & 0.40 & 250.20 & 6 & $<0.001$ \\
Hs*Js & 0.33 & 342.38 & 6 & $<0.001$ \\
Jh*Js & 0.79 & 39.80 & 6 & $<0.001$ \\
\hline
\end{tabular}

nella xenodactyloides (Blackburn \& Moreau 2006) fue de $0 \%$ en ambos casos. Sin embargo, en Xenohyla truncata, una especie arborícola, el porcentaje de individuos sin alimento fue de $53 \%$ (da Silva et al. 1989).

De las 22 categorías de alimento encontradas en $C$. lineatus, las más importantes fueron las arañas, coleópteros, formícidos, isópodos, ortópteros, quilópodos y la materia vegetal. Los estudios llevados a cabo en anuros postmetamórficos han documentado que los artrópodos son el recurso alimenticio principal de este grupo de vertebrados (Zug \& Zug 1979, Toft 1980a, 1980b \& 1985, Donelly 1991, Lima \& Moreira 1993, Duellman \& Trueb 1994, dos Santos et al. 2003; Santos et al. 2004, Mendoza-Estrada et al. 2008). Asimismo, las categorías encontradas son similares a las reportadas en otras ranas que viven en el suelo del bosque tropical como $E$. johnstonei, E. ockendeni, E. toftae, E. peruvianus, Edalorhina perezi y Leptodactylus andreae (Ovaska 1991, Toft 1981).

El consumo de materia vegetal por parte de $C$. lineatus posiblemente no sea un fenómeno accidental como algunos autores han sugerido para otras ranas, ya que estudios en Lithobates zweifeli, Rhinella marina y Pleurodema diplolistris han regis- 
trado un valor de ingesta importante de este recurso (Zug \& Zug 1979, dos Santos et al. 2003, Santos et al. 2004, Mendoza-Estrada et al. 2008). Asimismo, se ha documentado que Xenohyla truncata ingiere frutos intencionalmente (da Silva et al. 1989, da Silva \& de Britto-Pereira 2006). Mendoza-Estrada et al. (2008) proponen que la materia vegetal ayudaría a la fermentación del alimento, como ocurre en reptiles herbívoros. Sin embargo, hace falta investigar el papel real que tiene este recurso en el balance nutritivo de los anuros. Pues existen casos como el de $X$. truncata, que puede vivir en cautividad con una dieta exclusivamente de frutos (da Silva et al. 1989). Asimismo, da Silva \& de Britto-Pereira (2006) sugieren que el consumo de plantas también pudiera estar funcionando como un mecanismo para secuestrar toxinas, como sucede en algunas especies de anuros que ingieren exclusivamente artrópodos (Daly et al. 2000).

El tamaño de la rana puede explicar la presencia de algunas categorías de alimento, en relación con la distribución del tamaño y abundancia del recurso (Toft 1981, Lima \& Magnusson 2000). Por ejemplo, Lima (1998) encontró que en la hojarasca de la selva amazónica los ácaros y colémbolos fueron las presas pequeñas más abundantes, las hormigas, termitas y escarabajos (presas de talla mediana) representaron la siguiente categoría de abundancia, mientras que arañas, lepidópteros y ortópteros (las presas más grandes) fueron las menos abundantes. Tomando como referencia estos resultados encontramos que los juveniles de $C$. lineatus consumieron principalmente coleópteros y formícidos, presas de talla mediana, mientras que los adultos consumieron arañas y ortópteros, presas grandes. Estos resultados eran de esperar, si consideramos que el tamaño del cuerpo de la rana es un indicador del tamaño máximo de la presa que esta consume, tal como ocurre en Ceratophrys cornuta (Duellman \& Lizana 1994), Colostethus stepheni (Lima \& Moreira 1993); Elutherodactylus johnstonei (Ovaska 1991), Pseudacris triseriata (Christian 1982) y Leptodactylus ocellatus (Maneyro et al. 2004).

Los juveniles de $C$. lineatus consumieron mayor número de presas que los adultos, posiblemente para satisfacer su demanda energética y ante la limitación mecánica para consumir presas más grandes. Otras especies que tuvieron comportamientos similares fueron C. cornuta (Duellman \& Lizana 1994); E. coqui (Woolbright \& Stewart 1987) y Leptodactylus ocellatus (Maneyro et al. 2004). Lo contrario ocurre en S. xenodactyloides (Blackburn \& Moreau 2006) y Dendrobates pumilio (Donelly 1991). En esta última especie, las hembras de mayor talla consumieron en promedio más presas (85.3), que los machos de talla mediana (64.5) y juveniles de talla pequeña (53.7). Sin embargo, debemos tomar en cuenta que las presas que ingirió esta especie corresponde a artrópodos pequeños (ácaros y colémbolos).

Además de la limitante mecánica del aparato trófico impuesta por el tamaño y diseño de la rana, Toft (1981) y Simon \& Toft (1991) sugieren que los cambios ontogenéticos de la dieta de las ranas también esta influenciada por el modo de forra- 
jeo. Sin embargo, Lima \& Magnusson (2000) no encontraron evidencias sólidas para generalizar esta suposición, ya que mientras en unas especies como E. fenestratus fue válida, en otras como Epipedobates femoralis lo fue parcialmente, en cambio en Colostethus marchesianus no se cumplió. También el consumo de hormigas fue relacionado por Toft (1981) con el modo de forrajeo, sin embargo, Ovaska (1991) considera que en algunas especies del género Eleutherodactylus éste hábito se debe al hecho de ser nocturnas. Nosotros consideramos que el consumo de hormigas está más relacionado con el tamaño del depredador. Por ejemplo, encontramos que los juveniles de $C$. lineatus (LHC promedio $=16.50 \mathrm{~mm}$ ) consumieron más hormigas $(12 \%)$ en comparación con los machos adultos ( $\mathrm{LHC}=26.68 \mathrm{~mm} ; 8.3 \%)$, mientras que las hembras no usaron este recurso. Asimismo, los juveniles de C. cornuta (LHC $=26.3 \mathrm{~mm}$ ) consumieron más hormigas $(91 \%)$ que los subadultos $(\mathrm{LHC}>47.6 \mathrm{~mm})$ o hembras adultas $(40 \%)$. En cambio los juveniles de $S$. xenodactyloides (LHC $=$ 14.9-16.9 mm) y D. pumilio $(\mathrm{LHC}=19.0-22.0 \mathrm{~mm})$ consumieron menos hormigas que los adultos ( $8.3 \%$ y $74 \%$ para la primer especie; $39 \%$ y $56 \%$ para la segunda) (Blackburn \& Moreau 2006, Donelly 1989). Con base en estos datos, notamos una tendencia al mayor consumo de hormigas por ranas de talla mediana, con una LHC alrededor de los 15-27 mm, mientras que la ingesta disminuye hacia ambos extremos. A reserva de una exploración más profunda, el tamaño óptimo del depredador parece ser fundamental en el consumo de este recurso.

Las diferencias estaciónales encontradas en $C$. lineatus se debieron al consumo exclusivo de algunas categorías de alimento en determinada época, así como al porcentaje diferencial de las categorías más importantes, que estuvieron siempre presentes. Por ejemplo, encontramos que las ranas consumieron más arañas y ortópteros durante la época seca, mientras que durante la época húmeda las categorías preferidas fueron los coleópteros, formícidos, isópodos y materia vegetal. Estas diferencias pueden relacionarse con la variación que experimentan las poblaciones de artrópodos a lo largo del año en un ambiente estacional. Pues se ha documentado el efecto que la humedad, precipitación y temperatura, así como la producción de hojarasca, tienen sobre las poblaciones de la fauna del suelo (Toft 1980b, Brown et al. 2001). En este sentido Toft (980b) encontró que la mayor diversidad y abundancia de artrópodos en el piso de un bosque tropical ocurrió durante la época seca, mientras que en la época húmeda fue menor debido a la mayor pudrición de la hojarasca. De manera que la mayor cantidad de categorías de alimento encontradas en $C$. lineatus durante la época seca, puede ser reflejo de la mayor disponibilidad de presas que hay en su ambiente.

En conclusión la dieta de una muestra poblacional de $C$. lineatus en Río Negro, al Norte de Coapilla, Chiapas, se integró por 22 categorías de alimento, tales como arañas, coleópteros, himenópteros formícidos, isópodos, ortópteros, quilópodos y materia vegetal como las más importantes. Esta dieta fue similar a la descrita para otros anuros que viven en el piso de los bosques tropicales, de las familias Eleuthero- 
dactylidae y Leptodactylidae. Las diferencias ontogenéticas encontradas parecieron estar relacionadas con la incapacidad de la rana para acceder a presas de mayor talla, debido a la limitación morfológica impuesta por su aparato trófico. En tanto que las diferencias estaciónales parecieron estar relacionadas con la disponibilidad temporal del alimento.

AGRADECIMIENTOS. Al Dr. Alberto González Romero y aun un revisor anónimo cuyas observaciones permitieron mejorar el manuscrito original. Nuestro agradecimiento para Abenamar Pozo, Freddy Chanona, Octavio Brindis y Roberto Vidal por el apoyo brindado durante las recolectas de vertebrados en Coapilla. Al Dr. Fredi Penagos entonces director de la Escuela de Biología del UNICACH por su apoyo al proyecto "Vertebrados de Coapilla", del cual se deriva el presente trabajo. Al Dr. Raúl Muñiz Vélez† por su asesoría en la determinación taxonómica de los artrópodos. A los biólogos Evelyn Lazcano, Xazmín Morales e Isaías López por sus comentarios a las versiones preliminares del presente escrito. Al Dr. Miguel Ángel Armella, jefe del Departamento de Biología de la UAM Iztapalapa por su apoyo al primer autor, para obtener el periodo sabático durante el cual se redactó el presente trabajo.

\section{LITERATURA CITADA}

Beard, K. H., K. A. Vogt \& A. Kulmatiski. 2002. Top-down effects of a terrestrial frog on forest nutrient dynamics. Oecologia, 133: 583-593.

Beard, K. H., A. K. Eschtruth, K. A. Vogt, D. J. Vogt \& F. N. Scatena. 2003. The effects of the frog Eleutherodactylus coqui on invertebrates and ecosystem processes at two scales in the Luquillo Experimental Forest, Puerto Rico. Journal of Tropical Ecology, 19: 607-617.

Blackburn, D. C. \& C. S. Moreau. 2006. Ontogenetic diet change in the arthroleptid frog Schoutedenella xenodactyloides. Journal of Herpetology, 40: 388-394.

Boquimpani-Freitas, L., C. F. D. Rocha \& M. Van Sluys. 2002. Ecology of the horned leaf-frog, Proceratophrys appendiculata (Leptodactylidae), in an insular Atlantic rain-forest of southeastern Brazil. Journal of Herpetology, 36: 318-322.

Borror, D. J., Ch. A. Triplehorn \& N. F. Johnson.1989. An introduction to the study of insects. $6^{\text {th }}$ Ed. Saunders Coll. Publ. USA.

Brower, J. E., J. H. Zar \& C. N. von Ende. 1989. Field and laboratory methods for general ecology. 3rd Ed., Wm. C. Brown Publishers, USA.

Brown, G. G., C. Fragoso, I. Barois, P. Rojas, J. C. Patrón, J. Bueno, A. G. Moreno, P. Lavelle, V. Ordaz \& C. Rodríguez. 2001. Diversidad y rol funcional de la macrofauna edáfica en los ecosistemas tropicales mexicanos. Acta Zoológica Mexicana (n.s.). Número Especial 1: 79-110.

Christian, K. A. 1982. Changes in the food niche during postmetamorphic ontogeny of the frog Pseudacris triseriata. Copeia, 1982: 73-80.

Chu, H. F. 1949. How to know the inmature insects. Wm. C. Brown Co. Publ.

Daly, J. W:, H. M. Garraffo, P. Jain, T. F. Spande, R. R. Snelling, C. Jaramillo \& A. S. Rand. 2000. Arthropod-frog connection: decahydroquinoline and pyrrolizidine alkaloids common to microsympatric myrmicine ants and dendrobatid frogs. Journal of Chemical Ecology, 26: 73-85.

da Silva, H. R. \& M. C. de Britto-Pereira. 2006. How much fruit do fruit-eating frogs eat? An investigation on the diet of Xenohyla truncata (Lissamphibia: Anura: Hylidae). Journal of Zoology, 270: 692-698.

da Silva, H. R., M. C. de Britto-Pereira \& U. Caramaschi. 1989. Frugivory and seed dispersal by Hyla truncata, a Neotropical treefrog. Copeia, 1989: 781-783. 
De la Rosa, J. S., A. Eboli M. \& M. Dávila S. 1989. Geología del Estado de Chiapas. Superintendencia Zona Sureste, CFE., México.

Donnelly, M. A. 1989. Reproductive phenology and age structure of Dendrobates pumilio in northeastern Costa Rica. Journal of Herpetology, 23: 362-367.

Donnelly, M. A. 1991. Feeding patterns of the strawberry poison frog Dendrobates pumilio (Anura: Dendrobatidae). Copeia, 1991: 723-730.

Dos Santos, J. W. A., R. P. Damasceno \& P. L. B. da Rocha. 2003. Feeding habits of the frog Pleurodema diplolistris (Anura, Leptodactylidae) in Quaternary sand dunes of the Middle Rio São Francisco, Bahia, Brazil. Phyllomedusa, 2: 83-92.

Duellman, W. E. 2001. The hylid frogs of Middle America. Vol. 1. Society for the Study of Amphibians and Reptiles, USA.

Duellman, W. E. \& M. Lizana. 1994. Biology of a sit-and-wait predator, the Leptodactylid frog $\mathrm{Ce}$ ratophrys cornuta. Herpetologica, 50: 51-64.

Duellman, W. E. \& L. Trueb. 1994. Biology of amphibians. The Johns Hopkins University Press, USA.

Krebs, C. J. 1999. Ecological Methodology. $2^{\text {nd }}$. ed. Benjamin Cummings. Addison Wesley Longman Inc., CA, USA.

Lima, A. P. 1998. The effects of size on the diets of six sympatric species of postmetamorphic litter anurans in Central Amazonia. Journal of Herpetology, 32: 392-399.

Lima, A. P. \& W. E. Magnusson. 2000. Does foraging activity change with ontogeny? An assessment for six sympatric species of postmetamorphic litter anurans in Central Amazonia. Journal of Herpetology, 34: 192-200.

Lima, A. P. \& G. Moreira. 1993. Effects of prey size and foraging mode on the ontogenetic change in feeding niche of Colosthethus stepheni (Anura: Dendrobatidae). Oecologia, 95: 93-102.

Maneyro, R., D. E. Naya, I. da Rosa, A. Canavero \& A. Camargo. 2004. Diet of the South American frog Leptodactylus ocellatus (Anura, Leptodactylidae) in Uruguay. Iheringia, Série Zoología, 94: 57-61.

Mendoza-Estrada, L. J., R. Lara López \& R. Castro-Franco. 2008. Dieta de Lithobates zweifeli Hillis, Frost \& Webb, 1984 (Anura: Ranidae) en un río estacional del centro de México. Acta Zoológica Mexicana (n.s.), 24: 169-197.

Ovaska, K.1991. Diet of the frog Eleutherodactylus johnstonei (Leptodactylidae) in Barbados, West Indies. Journal of Herpetology, 25: 486-488.

Pianka, E. R. 1978. Evolutionary Ecology. Harper and Row, USA.

Santos, E. M., A. V. Almeida \& S. Vasconcelos. 2004. Feeding habits of six anuran (Amphibia: Anura) species in a rainforest fragment in Northeastern Brazil. Iheringia, Série Zoología, 94: 433-438.

Santos-Barrera, G., M. Acevedo, A. Muñoz Alonso. 2004. Craugastor lineatus. In: IUCN 2010. IUCN Red List of Threatened Species. Version 2010.4. <www.iucnredlist.org>. Downloaded on 28 December 2010.

Savage, J. M.1987. Systematics and distribution of the Mexican and Central American rain frogs of the Eleutherodactylus gollmeri group (Amphibia: Leptodactylidae). Fieldiana Zoology. N. S., 33: 1-57.

Simon, M. P. \& C. A. Toft. 1991. Diet specialization in small vertebrates: mite-eating in frogs. Oikos, 61: 263-278.

Taigen, L. T. \& F. H. Pough. 1983. Prey preference, foraging behavior, and metabolic characteristics of frogs. The American Naturalist, 122: 509-520.

Toft, C. A. 1980a. Feeding ecology of thirteen syntopic species of anurans in a seasonal tropical environment. Oecologia, 45: 131-141. 
Toft, C. A. 1980 b. Seasonal variation in populations of Panamanian litters frogs and their prey: a comparison of wetter and drier sites. Oecologia, 47: 34-38.

Toft, C. A. 1981. Feeding ecology of Panamanian litter anurans: patterns in diet and foraging mode. Journal of Herpetology, 15: 139-144.

Toft, C. A. 1985. Resource partitioning in amphibians and reptiles. Copeia, 1985: 1-21.

Zar, J. H. 1996. Biostatistical Analysis. 3rd. ed. Prentice-Hall, Inc., Upper Saddle River, NJ, USA.

Zug, G. R. \& Zug. 1979. The marine toad, Bufo marinus: a natural history résumé of native populations. Smithsonian Contribution to Zoology, 284: 1-58.

Zuill, H. A. \& E. W. Lathrop. 1975. The structure and climate of a tropical montane rain forest and an associated temperate pine-oak-liquidambar forest in the northern highlands of Chiapas, México. Anales del Instituto de Biología, UNAM, México 46, Serie. Botánica: 73-118. 